International Journal of Bifurcation and Chaos, Vol. 19, No. 8 (2009) 2695-2703

(c) World Scientific Publishing Company

\title{
HYSTERESIS AND VORTICES DYNAMICS IN A TURBULENT FLOW
}

\author{
JAVIER BURGUETE and ALBERTO DE LA TORRE \\ Departamento de Física y Matemática Aplicada, \\ Universidad de Navarra, \\ Irunlarrea 1, 31008 Pamplona, Spain
}

Received April 4, 2008; Revised October 2, 2008

\begin{abstract}
Recent results about the slow dynamics present in a fully developed turbulent flow are reported. In a previous paper [de la Torre \& Burguete, 2007] we showed that the mean velocity field in a turbulent flow bifurcates subcritically breaking some symmetries of the problem and becomes time-dependent because of equatorial vortices moving with a precession movement. This subcriticality produces a bistable regime, whose main characteristics were successfully reproduced using a three-well potential model with additive noise. In this paper we present the characterization of the hysteresis region, not previously observed, in this bifurcation. This hysteresis appears only for an extremely small range of parameters.
\end{abstract}

Keywords: Turbulent flow; subcritical transition; stochastic model; von Kármán swirling flow.

\section{Introduction}

One hundred and twenty five years after the works of O. Reynolds on the transition to turbulence, this regime still astonishes the scientific community with new results and possibilities [Jansson, 2006; Vatistas, 1990]. The fact is that, due to the nonlinearity of the Navier-Stokes equation no general solution has been found, and no theoretical nor numerical study can face the fully developed turbulent regime without strong restrictions or approximations. In this regime, only experimental observations of the flow can help us understand how nature works.

One of these experiments that have been widely studied is the flow that appears inside a closed cylindrical cavity, whose top and bottom can rotate independently (called a von Kármán flow) [von Kármán, 1921; Zandbergen, 1987]. After the proposal of this flow as a suitable candidate for the development of the dynamo instability [Odier, 1998] several numerical [Nore, 2003; Nore, 2006; Shew, 2006] and experimental studies [Ravelet, 2005; Ravelet, 2008; Marié, 2003; Nore, 2005] have been done.
The particular configuration we have studied uses two different propellers which counter rotate. Comparable flows have been optimized in similar configurations to maximize the magnetohydrodynamics (MHD) response [Monchaux, 2007; Berhanu, 2007]. In this process some important features of the flow have been simplified to benefit the numerical simulations, for example, making the velocity field axisymmetric, or neglecting the effect of coherent structures (such as equatorial vortices). Although these numerical studies have been used to predict successfully the thresholds of the dynamo action [Gailitis, 2000; Stieglitz, 2001; Pétrélis, 2003], real flows can present slow dynamics compared to the magnetic diffusion time that cannot be neglected and should be taken into account in the numerical codes.

In this paper, we will focus on the slow dynamics that appears for very large Re numbers. The flow presents a nontrivial alternation between two states that breaks some symmetries of the problem. Our objective is the analysis and characterization 

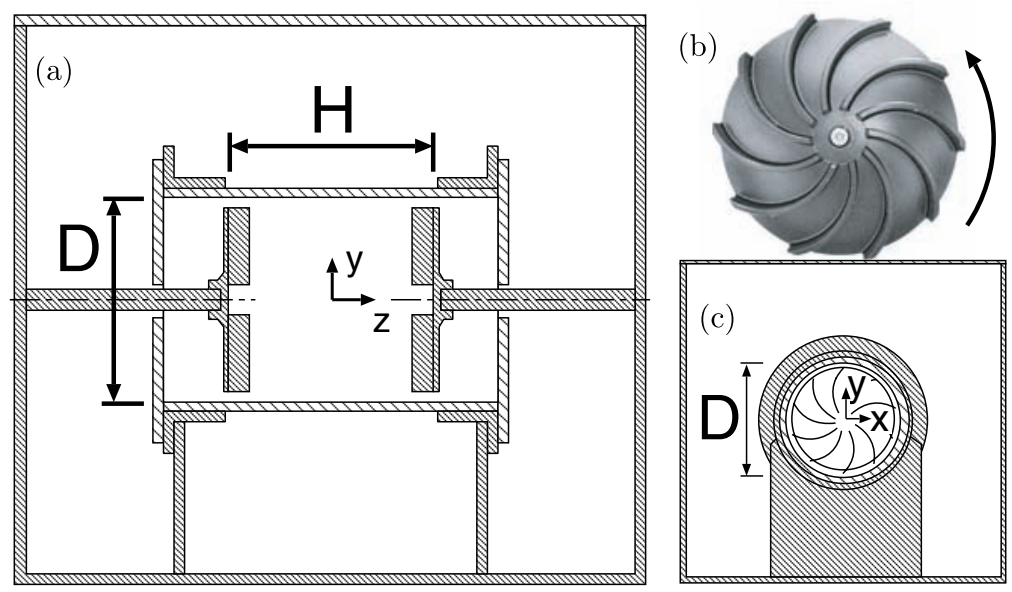

Fig. 1. Experimental setup: (a) Section along the axis of the cylinder. The cylinder is placed horizontally, inside a tank of $150 l$. (b) Propeller used in the experiment. The arrow indicates the rotation direction (positive in the south propeller). (c) View of the south propeller from the equatorial plane.

of the hysteresis region that appears in this dynamical system. A simple stochastic model can recover most of the experimental results, as the residence times, the exponential escape time and the forced response.

\section{Experimental Setup}

The experimental volume consists in a cylindrical vessel made of plexyglass (Fig. 1), whose top and bottom ends can rotate independently and modified at will. The aspect ratio $\Gamma=H / D$ can be modified continuously, and for the present results it has been fixed to $\Gamma=1$. In the configuration used in this paper, the propellers placed at both ends have 10 curved blades (blades' height, $2 \mathrm{~cm}$ ) that rotate pushing the fluid with the convex side. One of them, referred in this paper as "North", is placed at $z=H / 2$ and the other one, referred as "South", is placed at $z=-H / 2$. The container and propeller radii are, respectively, $R_{\text {cont }}=D / 2=10 \mathrm{~cm}$ and $R_{\text {prop }}=8.75 \mathrm{~cm}$. The experimental Reynolds number, based on the rim velocity, is $\operatorname{Re}=V_{\text {rim }} R_{\text {cyl }} / \nu=$ $2 \pi f_{\text {prop }} R_{\text {prop }} R_{\text {cyl }} / \nu$. In this paper we induce a dissymmetry in the experiment that will be controlled by a new parameter, the frequency dissymmetry, defined as $\Delta=\left(f_{N}-f_{S}\right) /\left(f_{N}+f_{S}\right)$, where $f_{N}$ and $f_{S}$ are the frequencies of each propeller, north and south.

The propellers are identical (within a $10 \mu \mathrm{m}$ precision) and we have taken special care to build an experimental setup as symmetric as possible. The geometry of the propellers has been chosen because of two reasons. First, it allows a high efficiency in terms of mean flow velocity compared to rim's velocity. With other geometries, i.e. flat discs, this efficiency can be as low as $1 / 20$, whereas in this configuration is close to one. The second reason is that this experiment has been conceived to study the potential application of this flow in a dynamo experiment. For this application, the flow needs a balance between the different velocity components that is achieved using these propellers (see for example [de la Torre, 2007b] and references within).

The measurement technique is a classical LDV velocimetry. With this technique, we can record extremely long time series (about $\sim 10^{5}$ rotation periods) of the velocity field, that allow a full characterization of the slow dynamics of the vortices present in the experiments. Using these data, the mean flow is obtained averaging the instantaneous flow for at least $10^{2}$ propeller turns.

For this study, the short- and long-term stability of the propeller velocities are very important. We have analyzed this variable using two techniques, one of them based on the propeller rim's velocity measurement using the LDV system, and the other one using a tachometer acting on the transmission system between the propeller and the motor. Both tools lead to the same conclusion: the mean velocity is stable, both in the short and long terms, with no appreciable drift within our experimental resolution (below one part in ten thousand). The fluctuations are random, normally distributed, and remain below a $0.07 \%$ when compared to the mean velocity.

The fluid used in this experiment is water, and the experimental Reynolds number can be varied 
in the range $\operatorname{Re}=10^{3}-10^{5}$. It is important to note that Reynolds numbers below $10^{3}$ cannot be reached with the present configuration. Details of this experimental setup can be found in [de la Torre, 2007a].

\section{Results and Discussion}

Prior to the new results, we present the key results obtained previously [de la Torre, 2007a], and that are needed in this context. For all the range of parameter values explored, the flow is in a fullydeveloped turbulent regime. Here we will focus on the situation where $\mathrm{Re}=3 \times 10^{5}$. In this situation, the flow is not steady, but fluctuates around an averaged state. The mean flow $\langle u\rangle=\left(\left\langle u_{r}\right\rangle,\left\langle u_{\theta}\right\rangle,\left\langle u_{z}\right\rangle\right)$ represented in Fig. 2 is obtained with average velocity series being longer than 300 times the period of the propeller. This flow is divided into two toroidal cells, each of them following the nearest propeller (positive azimuthal velocity in the south, negative in the north). In each cell, the fluid is aspired through the axis towards the propellers, where it is ejected to the walls. The flow returns along the cylinder's wall and the equatorial plane. Concerning fluctuations, the turbulence rate (rms value over the mean value) varies between $60-150 \%$.

As it can be observed in Fig. 2, this mean flow does not preserve the symmetry around the equator (a $\pi$ rotation around any axis in the $z=0$ plane, i.e. $R_{\pi}$ symmetry). In the case presented, the cell near the north propeller is bigger than the south one. The broken symmetry is recovered when the mirror state (south cell bigger than the north cell) is considered. Each state (labeled as "north" $N$ or "south" $S$ depending on which cell is the dominant one, i.e. bigger) are equally accessible when $\Delta=0$. This dissymmetry is in contradiction with other works [Ravelet, 2005, 2008] in which the $R_{\pi}$ symmetry is observed (i.e. the frontier between the two rolls is always in the plane $z=0$ ). Other experiments at much lower Reynolds numbers [Nore, 2005 ] $(\operatorname{Re} \leq 600)$ have shown that the axisymmetry can be broken producing near heteroclinic orbits, but preserving the $R_{\pi}$ symmetry.

When larger tracers (air bubbles) are used, coherent structures as vortices are visualized. These vortices have a characteristic size $D_{\text {vortex }}=5 \mathrm{~cm}$
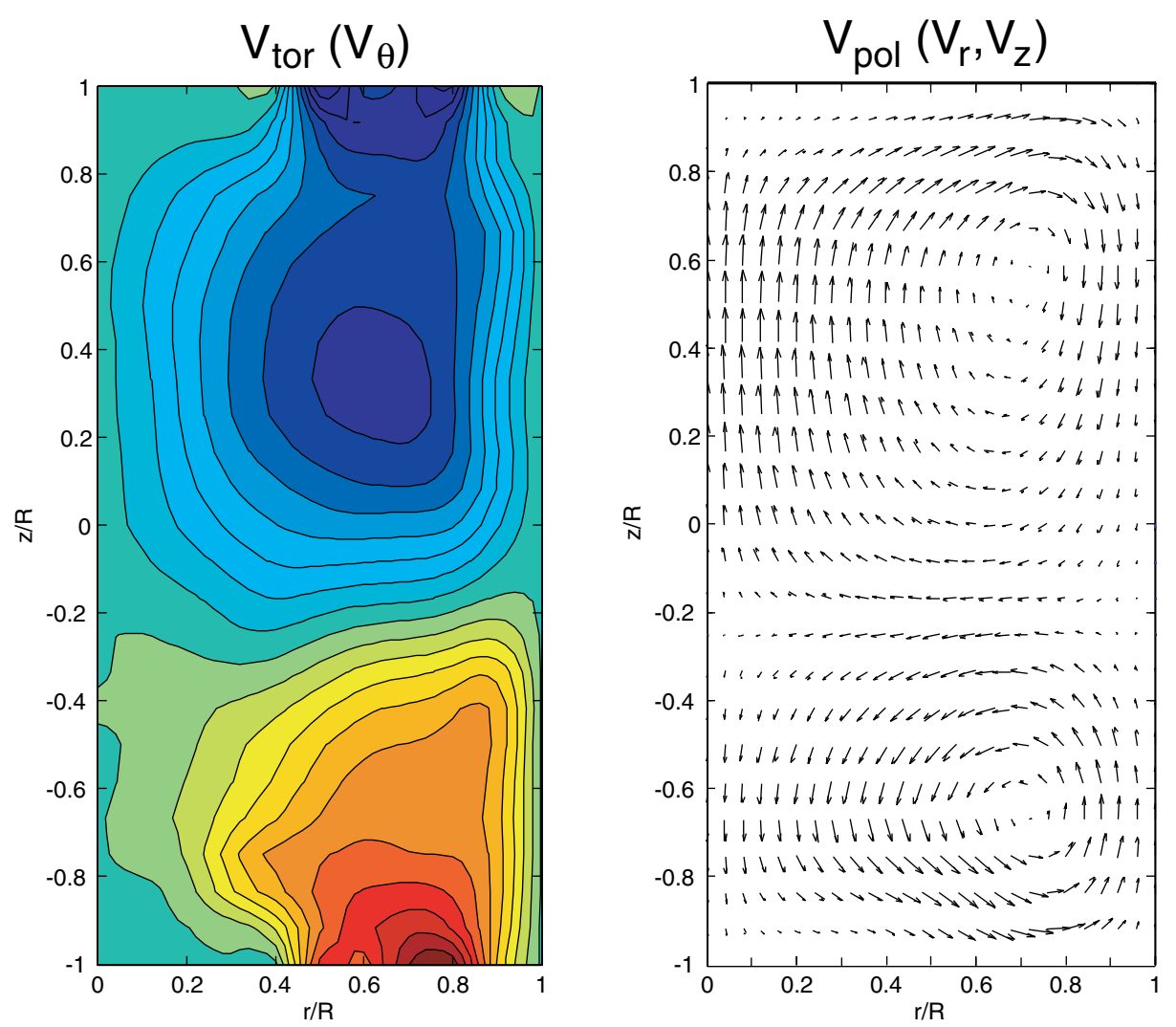

Fig. 2. (Left) Contourplot of $u_{\theta}$ (toroidal component). The blue and red colors mean respectively negative and positive azimuthal velocities. (Right) Stream-vectors $u_{r}, u_{z}$ in the plane $\theta=0$ (poloidal component). 
and appear simultaneously to the dissymmetry of the mean flow. Previous works in similar configurations [Nore, 2003] showed the formation of a static vortex in the equatorial plane $(z=0)$ for much lower Re, inaccessible with the present configuration.

The state $N$ or $S$ of the system can be characterized through the azimuthal velocity $u_{\theta}^{\mathrm{eq}}$ in an equatorial point near the wall $(r=0.9 R, z=0)$. This quantity fluctuates around an average value (in Fig. $2,\left\langle u_{\theta}^{\mathrm{eq}}\right\rangle \sim-0.4 \mathrm{~m} / \mathrm{s}$ ) with a turbulent rate that approaches $100 \%$.

A key point is that the averaged value obtained depends strongly on the period of time used to obtain this average. For extremely long periods of time (i.e. larger than $10^{4} \mathrm{~s}$ ), this averaged value is null, but a first look to the temporal evolution shows that the real behavior is not so simple: the system can be in any of two different states (see Fig. 3, top), each one with opposite average velocity. These two values of the azimuthal velocity correspond to the two asymmetric states previously described as "North" and "South". When this average is computed for very long periods of time, the system evolves jumping between the states North and South, recovering a perfectly symmetric (around the equator) velocity field.

The probability density function (PDF) of these states can be computed breaking up the data series in time intervals: a low pass filter is applied that allows to differentiate between $N$ and $S$ and split the signal (see Fig. 3, top). The shape of these PDFs does not depend on the cutoff frequency except for extreme values $\left(f_{\text {cut }} \rightarrow 0\right.$ or $f_{\text {cut }} \rightarrow$ sampling rate). In Fig. 3 (bottom) the PDF of the instantaneous azimuthal velocity $\left(u_{\theta}\right)$ for the two states are shown: north (with negative most probable $u_{\theta}$ ) and south (with positive most probable velocity). Inside these time intervals, and for each state, the average velocity can be computed. The absolute value is the same in both states, it is stable inside each interval (that can range from some hundreds seconds to various thousands) and proportional to the propeller's rim velocity. The typical transition time in a jump is about $10 \mathrm{~s}$, while the time between inversions can vary from minutes to hours. These time scales are much slower than the period of the propeller $\left(t_{\text {acq }}=2.4 \times 10^{5} T_{\text {prop }}\right.$, with $T_{\text {prop }}=1 /$ $\left.f_{\text {prop }}=0.2 \mathrm{~s}\right)$.

The analysis of the PDFs shows that these distributions are not gaussians. Nevertheless, each one of them $\left(p_{N}\right.$ or $\left.p_{S}\right)$ can be described as the superposition of two gaussians:

$$
\begin{aligned}
p_{N, S}\left(u_{\theta}\right)= & G_{0}+G_{N, S}=\frac{A_{0}}{\sqrt{2 \pi} \sigma_{0}} \exp \left(-\frac{u_{\theta}^{2}}{2 \sigma_{0}^{2}}\right) \\
& +\frac{A_{N, S}}{\sqrt{2 \pi} \sigma_{N, S}} \exp \left(-\frac{\left(u_{\theta}-u_{N, S}\right)^{2}}{2 \sigma_{N, S}^{2}}\right)
\end{aligned}
$$

with $A_{0}+A_{N, S}=1$.

Both distributions $p_{N}$ and $p_{S}$ share the same properties: one of the gaussians $G_{0}$ has zero mean, while the other $G_{N, S}$ is centered around a finite value $\left(\left|u_{N}\right|=\left|u_{S}\right| \neq 0\right)$ that increases slightly with the Re number. A more detailed discussion about the dependence of this results on the Reynolds number, and a discussion about their behavior can be found in [de la Torre, 2007a].

According to this description, the system visits three different regions in phase space around $u_{\theta}=$ $\left[0, u_{N}, u_{S}\right]$. A simple model based on a three-well potential (one for the symmetric case $u_{\theta}=0$ and the other two for the asymmetric states $u_{N, S}$ ) will describe this dynamics:

$$
\dot{u}_{\theta}=\epsilon u_{\theta}+g u_{\theta}^{3}-u_{\theta}^{5}+\sqrt{2 B} \xi(t)
$$

where $\xi(t)$ is a noise distribution with noise level $B$ (playing the role of the turbulence rate) and $g$ controls the relative depth of the potential wells. The $N$ state (resp. $S$ ) will appear when the system is wandering between the wells $u_{0}$ and $u_{N}$ (resp. $u_{0}$ and $\left.u_{S}\right)$. The parameter $\epsilon$ is varied in the range $\left(-g^{2} / 4<\epsilon<0\right)$ where the three solutions $u_{\theta}=$ $\left[0, \pm\left(g / 2+\left(g^{2} / 4+\epsilon\right)^{.5}\right)^{.5}\right]$ are stable.

Different runs using an Euler-Maruyama scheme [Kloeden, 1999] were performed in order to recover the dynamics: for small $\epsilon$ the dynamics is confined to the region around $u_{\theta}=0$ whereas for $\epsilon \rightarrow 0$ the numerical evolution presents spontaneous inversions. In this latter case the PDF of each state can be computed and compared to experiments. The characteristic doubly bumped distribution is obtained, but in the numerical distribution the queues are not symmetric due to the shape of the sixth order potential in the neighborhood of $u_{S, N}$.

The distribution of the times the system stays in one state (residence times) follows an exponential decay law (Kramer's escape rate [Kramers, 1940; Hänggi, 1990; Chechkin, 2005]):

$$
\rho(t)=\frac{1}{T_{0}} \exp \left(\frac{-t}{T_{0}}\right)
$$

where $T_{0}$ (escape time-scale, or escape time) is related to the intensity of noise. 

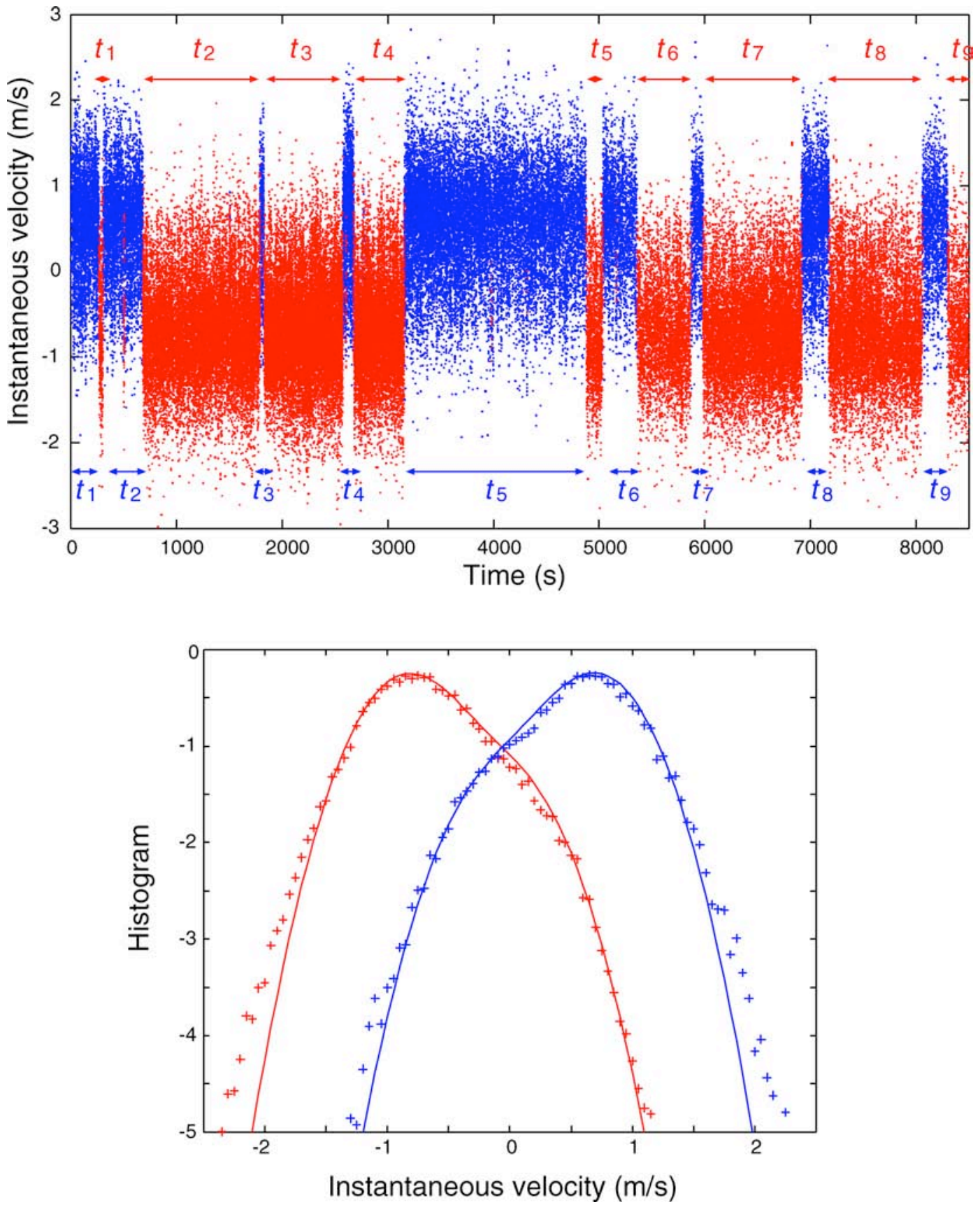

Fig. 3. (Top) Instantaneous azimuthal velocity temporal series. In order to distinguish between the two possible states, the spots have been colored, red for the north state (with negative predominant velocity) and blue for the south state (with velocities mainly positives). The duration of each interval has been labeled as $t_{i}$, with $i=1, \ldots, 9$, for each state. (Bottom) Histogram of the south (blue) and north (red) states. The whole set of intervals has been used to compute these PDFs. The crosses correspond to the experimental data. The continuous lines correspond to the best fit using Eq. (1) $\left(\sigma_{0}=\sigma_{N, S}\right.$, $\left.A_{0} \simeq 0.25, A_{N, S} \simeq 0.75\right)$.

When a dissymmetry is forced on the system, one of the states is favored, and the other is penalized. A very simple way to introduce this effect is the introduction of a "tilt" in the potential of Eq. (2):

$$
U=-\kappa u_{\theta} f(\Delta)-\frac{1}{2} \epsilon u_{\theta}^{2}-\frac{1}{4} g u_{\theta}^{4}+\frac{1}{6} u_{\theta}^{6}
$$

where $f(\Delta)$ reflects the dissymmetry and $\kappa$ is a positive parameter. For small $\Delta$, the dissymmetry term can be expanded in a Taylor series, and retaining only the first nontrivial term, the model in Eq. (2) reads (including the stochastic term) as:

$$
\dot{u}_{\theta}=\epsilon u_{\theta}+g u_{\theta}^{3}-u_{\theta}^{5}+\kappa \Delta+\sqrt{2 B} \xi(t)
$$





Fig. 4. Schema of the three-well potential used in Eqs. (2), (4) and (5) for different $\Delta$ values. The relative depth of each well depends on $\Delta$. The gray dashed line corresponds to the effect of the linear term in Eq. (4).

When $\Delta \neq 0$, a dissymmetry appears in the potential, but when $\Delta$ is small enough, it preserves the three-well system (see Fig. 4). For this range of $\Delta$, a hysteresis region is expected. On the other side, for large values of $\Delta$ one of the wells disappears, and only one of the states is preserved, as was obtained in [de la Torre, 2007a].

In the previous paper [de la Torre, 2007a] we stated that no inversions was observed with $\Delta \neq$ 0 , so up to our precision at that time and measurement times no hysteresis was found. With the improved experimental setup, we have access to smaller values of $\Delta$. Depending on the value of $\Delta$, one of the mixed states dominates (see Fig. 5). This behavior corresponds to a hysteresis region in Eq. (5). Without any noise, the system goes to one or the other states depending on the initial conditions and/or the perturbation of the system. Once the system is trapped in one of the wells, only a sufficiently large perturbation can make the system jump. When a significant noise is present, the system will switch from one state to the other, and back, for random periods of time.

For a perfectly symmetric problem, the distribution of residence times will be the same for the North- and South-states. But, when the symmetry is broken, one of these states will have a distribution with larger values of the corresponding residence times than those of the other distribution. According to the procedure described above we can slice the velocity series in sections with the same state. For each one of these sections, we can compute the different residence times (noted as $t_{i}$ in Fig. 3, red for the North state, and blue for the South state).

The distributions of these residence times can be computed, and we have checked that they effectively describe an exponential decay, as was

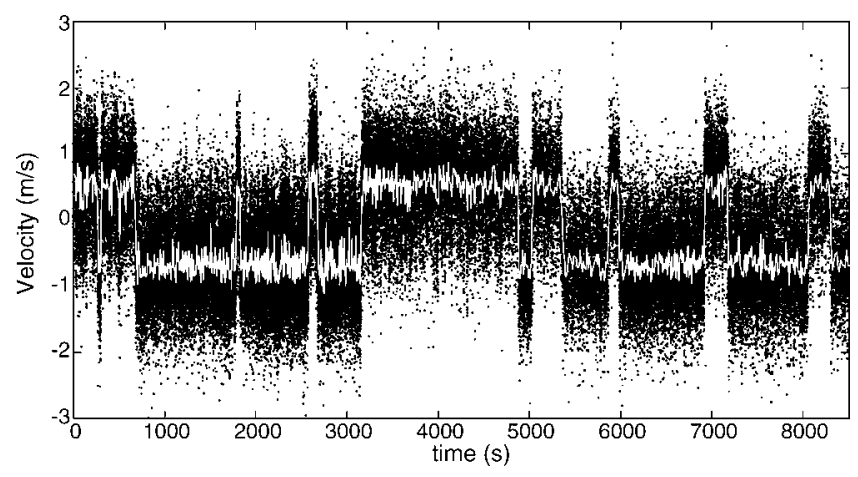

(a)

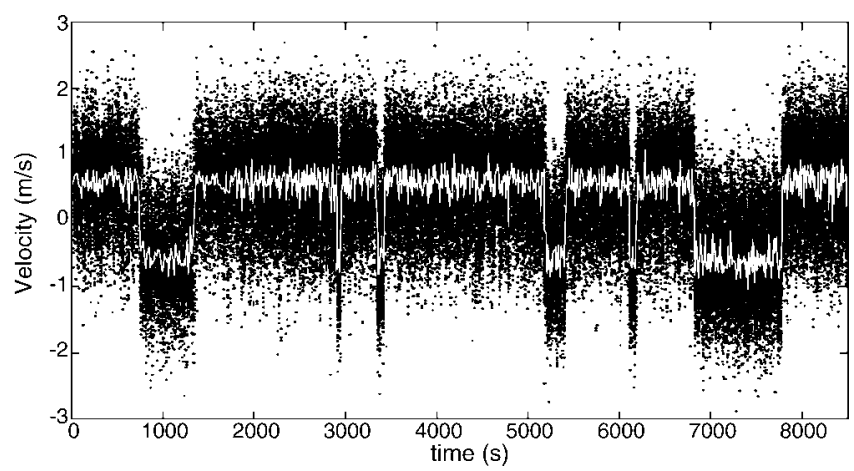

(b)

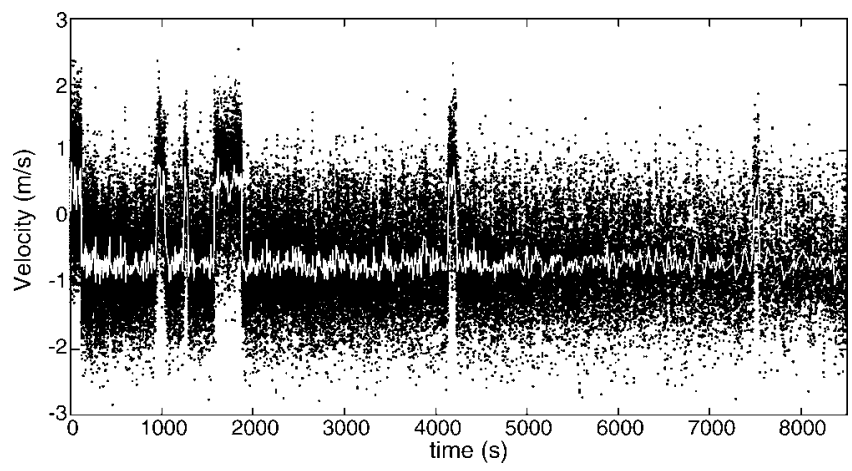

(c)

Fig. 5. Temporal evolution of the azimuthal velocity at $(r=0.9, z=0)$ for three different experimental situations: (a) $\Delta=0$, symmetric state, (b) $\Delta=+0.0017>0$, the so-called South-state dominates, and (c) $\Delta=-0.0017<0$ where the North-state is visited during longer periods of time. (The mean frequency of the propellers remains constant in the three data series: $\bar{f}=\left(f_{N}+f_{S}\right) / 2=7.76 \mathrm{~Hz}$. $)$

expected [Eq. (3)]. In Fig. 6 the corresponding distributions are presented for two different situations, $\Delta= \pm 0.0017$. When $\Delta<0$ (resp. $\Delta>0$ ), the North state has larger (resp. smaller) residence times than the South state, as it was expected. 


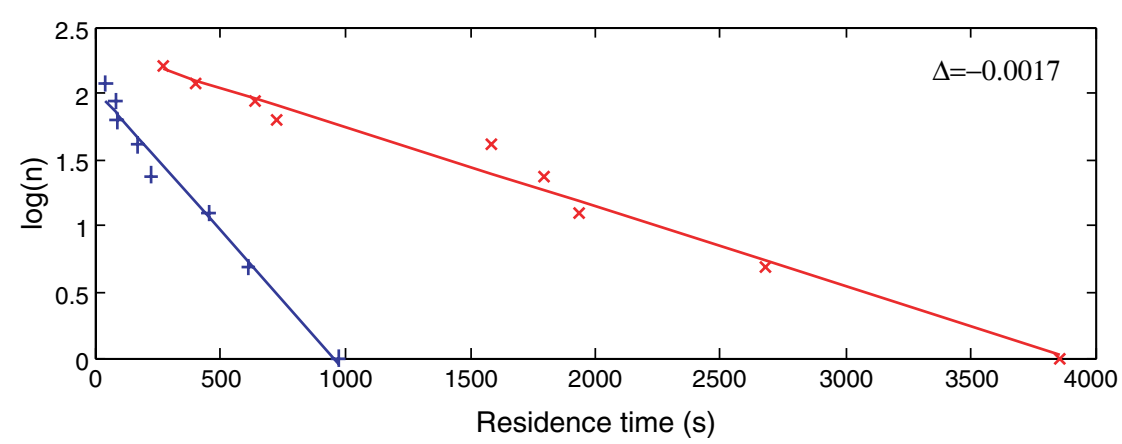

(a)

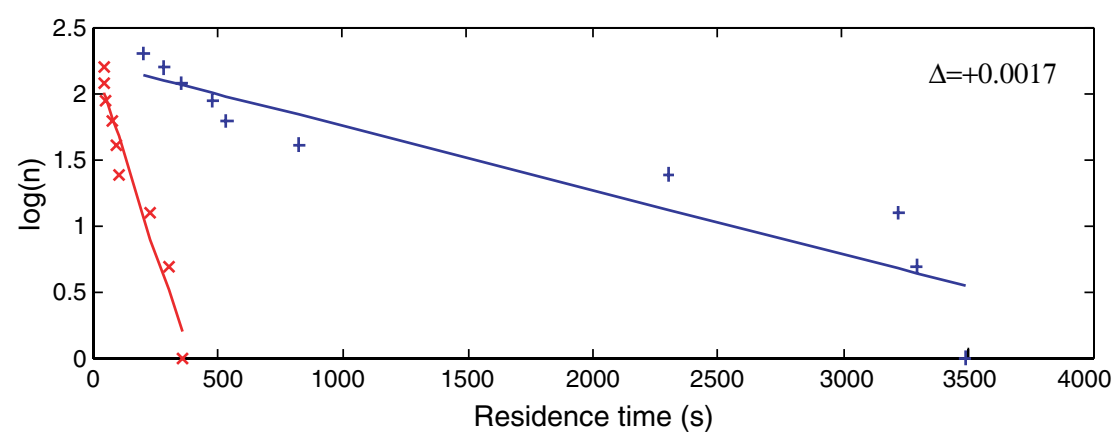

(b)

Fig. 6. Histogram (in logarithmic scale) of the residence times as defined in Fig. 3 for two different situations: (a) $\Delta=$ $-0.0017<0$, where the north state dominates, and consequently, has longer residence times, and (b) $\Delta=+0.0017>0$, where the south state dominates. As in other figures, the red symbols $(x)$ correspond to the north state, and the blue ones $(+)$ to the south state. The straight lines correspond to an exponential fitting using the Kramer's expression [Eq. (3)]. The slope of these lines is $-1 / T_{0}$, where $T_{0}$ is the $N, S$-state escape time.

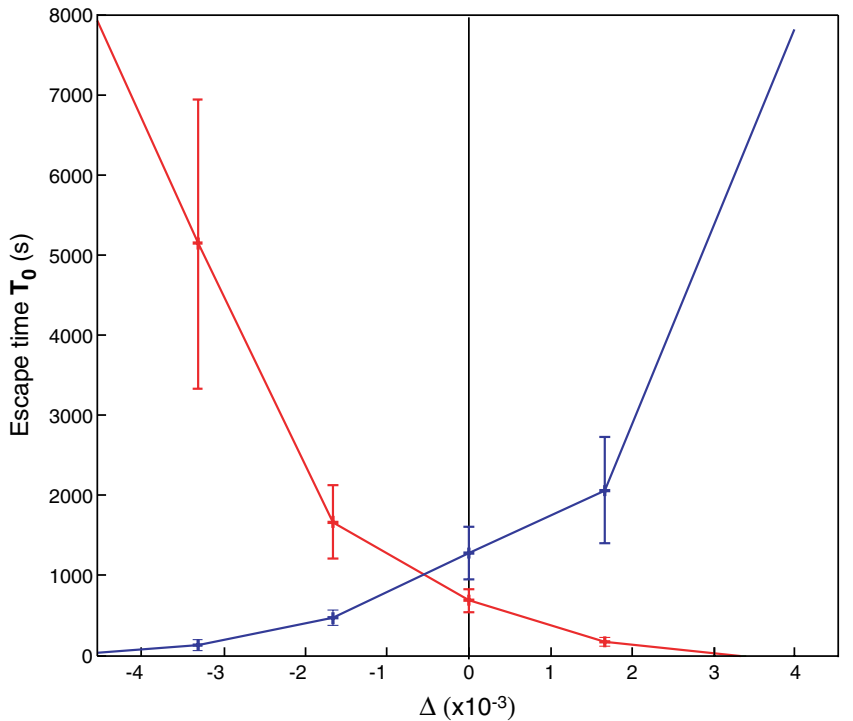

Fig. 7. Escape times $T_{0}$ for the two states $(N, S)$ as a function of the dissymmetry parameter $\Delta$. Large (resp. small) escape time means that the corresponding state remains for long (resp. short) times. Dashed lines mean that the corresponding points have been obtained with a very poor statistic (very few residence times have been used to construct the histogram) and should be considered as a tendency.
Using Eq. (3), we can obtain the escape time $T_{0}$ for each state and every $\Delta$ value. The results are summarized in Fig. 7, where it can be observed that a very precise experimental setup is necessary to obtain this hysteresis region. Only for $|\Delta| \lesssim 0.002$ escape times smaller than 5000s can be obtained, that is in the limit of most experimental runs. However, it should be noted that in this configuration there is a very small dissymmetry in the experimental setup, because both states have the same escape time for $\Delta \simeq-0.0005$.

It is important to note that this same setup, reducing the control on the propeller frequencies (for example, a natural fluctuation of $\Delta$ of 0.01 is allowed) will induce fast jumps between the North and South states that could produce, on average, a symmetric velocity field around the equator.

\section{Conclusions}

We have presented experimental evidence of the subcritical bifurcation of a turbulent flow, where some symmetries are broken. Two different 
asymmetrical states appear, and a bistability regime arises that initiates a complex and very rich dynamics. Using an external forcing, the system can be controlled and one of the states is favored to the detriment of the other. The hysteresis region has been determined, and the experimental residence times of each solution have been computed. These times obey an exponential distribution, and the corresponding escape times (Kramer escape rate) have been calculated.

A toy model has been introduced, that can successfully recover the characteristics of the dynamics. These results have been obtained using a highly precise experimental setup, where the propellers frequency stability is assured, up to a value of a $0.07 \%$. It is important to note that a less controlled experimental setup will induce fluctuations and timedependent asymmetries on an such a way that the broken symmetries can be recovered on an average.

\section{Acknowledgments}

This work has been partly supported by the Spanish Government through Contract No. FIS2007-66004C02-01 and by PIUNA (University of Navarra, Spain). A. de la Torre acknowledges financial support from the "Asociación de amigos de la Universidad de Navarra".

\section{References}

Berhanu, M. et al. [2007] "Magnetic field reversals in an experimental turbulent dynamo," Europhys. Lett. 77, 59001.

Bourgoin, M., Marié, L., Pétrélis, F., Burguete, J., Chiffaudel, A., Daviaud, F., Fauve, S., Odier, P. \& Pinton, J.-F. [2002] "MHD measurements in the von Kármán sodium experiment," Phys. Fluids 14, 3046-3058.

Chechkin, A., Gonchar, V., Klafter, J. \& Metzler, R. [2005] "Barrier crossing of a Lévy flight," Europhys. Lett. 72, 348 .

de la Torre, A. \& Burguete, J. [2007a] "Slow dynamics in a turbulent von Karman swirling flow," Phys. Rev. Lett. 99, 054101.

de la Torre, A. \& Burguete, J. [2007b] "Influence of time dependent flows on the threshold of the kinematic dynamo action," Eur. Phys. J.-ST. 146, 313.

Frisch, U. [1995] Turbulence - The Legacy of A. N. Kolmogorov (Cambridge Univ. Press).

Gailitis, A. et al. [2000] "Detection of a flow induced magnetic field eigenmode in the Riga dynamo facility," Phys. Rev. Lett. 84, 4365.

Hänggi, P. \& Talkner, P. [1990] "Reaction-rate theory: Fifty years after Kramers," Rev. Mod. Phys. 62, 251.
Holmes, P. et al. [1996] Turbulence, Coherent Struct. Dynamical Systems and Symmetry (Cambridge Univ. Press).

Jansson, T. R. N., Haspang, M. P., Jensen, K. H., Hersen, P. \& Bohr, T. [2006] "Polygons on a rotating fluid surface," Phys. Rev. Lett. 96, 174502.

Kloeden, P. E. \& Platen, E. [1999] Numerical Solution of Stochastic Differential Equations (Springer).

Kramers, H. [1940] "Brownian motion in a field of force and the diffusion model of chemical reactions," Physica $A$ 7, 284.

Marie, L., Burguete, J., Daviaud, F. \& Leorat, J. [2003] "Numerical study of homogeneous dynamo based on experimental von Karman type flows," Eur. Phys. J. $B$ 33, 469.

Moffatt, H. K. [1978] Magnetic Field Generation in Electrically Conducting Fluids (Cambridge University Press, Cambridge).

Monchaux, R. et al. [2007] "Generation of magnetic field by dynamo action in a turbulent flow of liquid sodium," Phys. Rev. Lett. 98, 044502.

Nore, C., Tuckerman, L. S., Daube, O. \& Xin, S. [2003] "The 1:2 mode interaction in exactly counter-rotating von Karman swirling flow," J. Fluid Mech. 477, 51.

Nore, C., Moisy, F. \& Quartier, L. [2005] "Experimental observation of near-heteroclinic cycles in the von Karman swirling flow," Phys. Fluids 17, 064103.

Nore, C., Witkowski, L. M., Foucault, E., Pecheux, J., Daube, O. \& Le Quere, P. [2006] "Competition between axisymmetric and three-dimensional patterns between exactly counter-rotating disks," Phys. Fluids 18, 054102.

Odier, P., Pinton, J. F. \& Fauve, S. [1998] "Advection of a magnetic field by a turbulent swirling flow," Phys. Rev. E 58, 7397-7401.

Pétrélis, F., Bourgoin, M., Marié, L., Burguete, J., Chiffaudel, A., Daviaud, F., Fauve, S., Odier, P. \& Pinton, J.-F. [2003] "Nonlinear magnetic induction by helical motion in a liquid sodium turbulent flow," Phys. Rev. Lett. 90, 174501.

Ravelet, F., Marie, L., Chiffaudel, A. \& Daviaud, F. [2004] "Multistability and memory effect in a highly turbulent flow: Experimental evidence for a global bifurcation," Phys. Rev. Lett. 93, 164501.

Ravelet, F., Chiffaudel, A., Daviaud, F. \& Leorat, J. [2005] "Toward an experimental von Karman dynamo: Numerical studies for an optimized design," Phys. Fluids 17, 117104.

Ravelet, F., Chiffaudel, A. \& Daviaud, F. [2008] "Supercritical transition to turbulence in an inertially-driven von Karman closed flow," J. Fluid Mech. 601, 339364.

Shen, W. Z., Norensen, J. \& Michelsen, J. [2006] "Numerical study of swirling flow in a cylinder with rotating top and bottom," Phys. Fluids 18, 064102. 
Stieglitz, R. \& Müller, U. [2001] "Experimental demonstration of a homogeneous two-scale dynamo," Phys. Fluids 13, 561.

Vatistas, G. H. [1990] "A note on liquid vortex sloshing and Kelvin's equilibria," J. Fluid Mech. 217, 241. von Kármán, T. [1921] "Über laminare und turbulente reibung," Z. Angew Math. Mech. 1, 233.

Zandbergen, P. \& Dijkstra, D. [1987] "Von Karman swirling flows," Ann. Rev. Fluid Mech. 19, 465. 